\title{
Recombinant or Urinary Human Chorionic Gonadotropin in Ovulation Induction?
}

\author{
Allahbadia Gautam
}

Published online: 17 January 2012

(C) Federation of Obstetric \& Gynecological Societies of India 2012

The onset of the spontaneous LH surge in women is relatively abrupt with concentrations doubling within $2 \mathrm{~h}$ to between 100 and $200 \mathrm{IU} / \mathrm{l}$ being sustained for 12-14 h over a mean duration of $50 \mathrm{~h}$ [1]. This mid-cycle surge, in addition to follicular rupture [2], promotes several periovulatory events including disruption of the oocytecumulus oophorus cell contact and induction of the resumption of the oocyte's meiotic maturation [3], cumulus oophorus mucification, luteinization of the follicular granulosa cells [4] and secretion of progesterone.

These events are also induced by an injection of human chorionic gonadotropin (hCG) through exactly the same sequence, the only difference being in the pharmacokinetic profile where the mean duration of the 'surge' after an IM injection of 5,000 IU u-hCG is longer than for $\mathrm{LH}(\sim 96 \mathrm{~h})$ and maximum concentrations may be less [5]. Current practice is that $\geq 5,000 \mathrm{IU} \mathrm{u}-\mathrm{hCG}$ is an acceptable

Allahbadia G. ( $\square)$, Medical Director

Rotunda Blue Fertility Clinic \& Keyhole Surgery Center,

Shivaji Park, Mumbai, India

e-mail: drallah@gmail.com

\section{Allahbadia G.}

Rotunda Fertility Clinic \& Keyhole Surgery Center, Lokhandwala, Andheri, Mumbai, India

Allahbadia G.

Rotunda-The Center for Human Reproduction, 36 Turner Road, 101, 1st Floor, B-Wing, Bandra (W), Mumbai, 400 050, India ovulatory dose and that lesser concentrations can lead to reduced oocyte recovery and a lower fertilization rate in assisted reproductive treatments [6].

The first preparations of hCG were investigated in the late $1930 \mathrm{~s}$ [7]. Subsequent studies and improved preparation of hCG from the urine of pregnant women (u-hCG), where its abundance allowed ease of extraction, meant that for almost 40 years it has been the sole hormonal preparation commercially marketed for induction of ovulation in anovulatory women. For the last three decades patients undergoing assisted reproductive treatments have also used $\mathrm{u}$-hCG to induce the final maturation of follicles and oocytes before their collection and additionally to support the luteal phase of the cycle.

The history of gonadotropin use when derived from either animal or human tissues has, however, not always been without clinical danger (e.g. antibody formation from pregnant mare serum gonadotropin; Creutzfeld-Jacob disease from human pituitary gonadotropin) so, as recombinant technology evolved, the logic of increasing both a compound's purity and safety could not be ignored. Such a uniform, specific product would mean that drug production would no longer be dependent on the vagaries of urine collection and hormone extraction, allowing commercial production to be adjusted according to market requirements. In addition all urinary contaminants would also be removed. Furthermore this would allow the safe subcutaneous administration of a compound with less batch-tobatch variation than has been demonstrated for urinary menopausal gonadotropins preparations [8]. 
Recombinant hCG (r-HCG) had been initially manufactured by transfecting non-human cell lines (Chinese hamster ovary cells) with genetic material capable of replicating identical amino acid sequences to the human compound and developed as a pharmaceutical product named Ovitrelle (Merck Serono, Switzerland). Today you have bio-similar molecules available in India (Triggerix, Lupin Ltd, India). However, prescribing a biosimilar to a patient calls for certain basic understanding by physicians of the scientific factors associated with the safety and efficacy of these products. Substituting an innovator brand by a biosimilar brand calls for caution in terms of quality, safety and efficacy aspects due to clear differences between biosimilars and their reference products [9].

A randomized, controlled, double-blind, double-dummy, phase III clinical trial was conducted in 84 women to compare the efficacy of a s.c. injection of $250 \mu \mathrm{g} \mathrm{r}-\mathrm{hCG}$ to an i.m. injection of 5,000 IU u-hCG in inducing folliculogenesis, resumption of oocyte meiosis and luteinization after ovulation induction with r-FSH. Since the confidence intervals for the difference of the number of oocytes retrieved between the two treatment groups were within the bounds defined by the multi-trial protocol, equivalence between r-hCG and u-hCG could be declared [10].

The International r-hCG Study Group conducted a Phase III, double-blind, double-dummy, randomized, parallelgroup, multicenter study to compare the safety and efficacy of $250 \mu \mathrm{g} \mathrm{r}-\mathrm{hCG}$ and 5,000 IU u-hCG, both administered s.c., for ovulation induction in anovulatory/oligo-ovulatory patients after follicular stimulation with r-FSH [11]. Subcutaneous $\mathrm{r}-\mathrm{hCG}$ and $\mathrm{u}-\mathrm{hCG}$ show equivalent efficacy in ovulation induction; however, $\mathrm{r}-\mathrm{hCG}$ is better tolerated [11].

The European Recombinant Human Chorionic Gonadotropin Study Group conducted a multicenter, doubleblind, double-dummy, randomized, parallel-group study comparing the efficacy and safety of r-hCG and u-hCG for inducing final follicular maturation and early luteinization in women undergoing ovulation induction for ART [12]. Following long down-regulation and stimulation with r-FSH, a total of 190 women received a single, SC injection of either $250 \mu \mathrm{g} \mathrm{r}-\mathrm{hCG}$ or $5,000 \mathrm{IU} \mathrm{u}-\mathrm{hCG}$. The mean number of mature oocytes was statistically higher $(P=0.027)$ for the $\mathrm{r}-\mathrm{hCG}$ group than the $\mathrm{u}-\mathrm{hCG}(9.4 \mathrm{vs}$. $7.1)$. Serum progesterone concentrations on day 1 and days 6-7 post-hCG, and serum hCG concentrations at all posthCG time points were statistically significantly in favor of r-hCG. The clinical pregnancy rate was somewhat higher (not significant) in the r-hCG group (33 vs. 25\%) as was the live birth rate ( 27 vs. $23 \%$, not significant). Both treatments were well tolerated, though the incidence of adverse events was significantly higher in the $\mathrm{u}-\mathrm{hCG}$ group (45.1 vs. $22.7 \%, P=0.0004)$. The incidence of injection-site reactions was significantly lower in the $\mathrm{r}-\mathrm{hCG}$ group $(P=0.0001)$. In conclusion, for triggering ovulation, r-hCG seems to have significant advantages compared with $\mathrm{u}-\mathrm{hCG}$ in terms of number of mature oocytes retrieved, luteal progesterone and local tolerance [12].

Farrag et al. conducted a prospective randomized study in order to investigate the effect of $\mathrm{r}-\mathrm{hCG}$ on oocyte nuclear and cytoplasm maturity compared to u-hCG, for inducing ovulation in women treated with ICSI for male factor infertility [13]. Their results showed that r-hCG increases the rate of metaphase II oocytes, the number and the rate of MII oocytes with mature cytoplasm compared to u-hCG [13].

An age-matched retrospective analysis compared the clinical outcomes of $\mathrm{r}-\mathrm{hCG}$ and $\mathrm{u}-\mathrm{hCG}$ in patients undergoing fresh, non-donor IVF cycles and concluded that $\mathrm{r}-\mathrm{hCG}$ was as effective as $\mathrm{u}-\mathrm{hCG}$ for final follicular maturation in IVF cycles [14].

A prospective, randomized and blinded comparison between 10,000 IU urinary and $250 \mu \mathrm{g}$ r-hCG for oocyte maturation in IVF cycles summarized that $\mathrm{r}-\mathrm{hCG}$ is at least as effective for inducing final stages of oocyte maturation as $10,000 \mathrm{IU} \mathrm{u}-\mathrm{hCG}$ and is also associated with significantly better patient tolerance and thus higher patient acceptability [15].

Kovacs et al. conducted a prospective randomized study between $250 \mu \mathrm{g}$ of r-hCG and 7,500 IU of u-hCG as the final trigger of ovulation during IVF [16]. They concluded that recombinant and urinary hCG provided similar serum and follicular hormonal environments during the final stages of oocyte maturation. The IVF outcome parameters were also comparable. The two medications appear to be equally effective [16].

Chan et al. set up a study to compare the effectiveness of 250 and $500 \mu \mathrm{g}$ r-hCG, which represented the lower and upper limits of the dose range, in inducing final oocyte maturation during IVF and ICSI cycles. The two doses of r-hCG were equally effective in inducing final oocyte maturation [17]. It remains unclear whether the higher midluteal estradiol and progesterone levels in the $500 \mu \mathrm{g}$ r-hCG group confer any benefit.

Littman and Milki described three cases in which the addition of r-hCG to $\mathrm{u}-\mathrm{hCG}$ to trigger ovulation in IVF improved oocyte recovery in patients with a history of scant oocyte yield in previous cycles [18].

\section{Conclusions}

The published data consistently show that single doses of $250 \mu \mathrm{g} \mathrm{r}-\mathrm{hCG}$ and 5,000 IU u-hCG produce similar clinical outcomes when used in infertility treatment cycles for timed intercourse, IUI, and IVF in terms of the number of 
oocytes retrieved, number of mature oocytes harvested, and fertilization and pregnancy rates attained $[8,10,12,13,17$, 19]. Single doses of $10,000 \mathrm{IU}$ u-hCG also gave results comparable to single doses of $250 \mu \mathrm{g} \mathrm{r}-\mathrm{hCG}$ [15]. $P$ levels in the midluteal phase were significantly higher with the use of r-hCG compared with u-hCG, and local injection site adverse effects were significantly less frequent, demonstrating the higher purity of the recombinant product [11, 12]. A single dose of $250 \mu \mathrm{g} r-\mathrm{hCG}$ was at least as effective as single doses of 5,000 or 10,000 IU u-hCG but offered the advantages associated with use of a recombinant product; local injection site adverse effects were significantly less frequent with r-hCG than with u-hCG [11-13, 17].

\section{References}

1. Hoff JD, Quigley ME, Yen SSC. Hormonal dynamics at midcycle: a revelation. J Clin Endocrinol Metab. 1983;57:792-5.

2. Espy LL. Ovarian proteolytic enzymes and ovulation. Biol Reprod. 1974;10:216-35.

3. Laurence TS, Dekel N, Beers WH. Binding of human chorionic gonadotropin by rat cumuli oophori and granulosa cells: a comparative study. Endocrinology. 1980;106:1114-8.

4. Brailly S, Gougeon A, Milgrom E. Androgens and progestins in the human ovarian follicle: differences in the evolution of preovulatory, healthy nonovulatory, and atretic follicles. J Clin Endocrinol Metab. 1981;53:128-34.

5. Fischer RA, Nakajama ST, Gibson M, et al. Ovulation after intravenous and intramuscular human chorionic gonadotrophin. Fertil Steril. 1993;60:418-22.

6. Abdalla HI, Ah-Moye M, Brinsden P. The effect of the dose of human chorionic gonadotrophin stimulation on oocyte recovery rates in an in vitro fertilisation program. Fertil Steril. 1987;48: 958-63.

7. Lunenfeld B, Insler V. From animal gonadotrophins to recombinant FSH. Perth: Ladybrook Publishing; 1999.

8. Ludwig M, Doody KJ, Doody KM. Use of recombinant human chorionic gonadotropin in ovulation induction. Fertil Steril. 2003; 79(5):1051-9.
9. Hincal F. An introduction to safety issues in biosimilars. Followon biopharmaceuticals. J Med CBR Def. 2009;7:1-17.

10. Driscoll GL, Tyler JP, Hangan JT, et al. A prospective, randomized, controlled, double-blind, double-dummy comparison of recombinant and urinary HCG for inducing oocyte maturation and follicular luteinization in ovarian stimulation. Hum Reprod. 2000;15(6):1305-10.

11. International Recombinant Human Chorionic Gonadotropin Study Group. Induction of ovulation in World Health Organization group II anovulatory women undergoing follicular stimulation with recombinant human follicle-stimulating hormone: a comparison of recombinant human chorionic gonadotropin (rhCG) and urinary hCG. Fertil Steril. 2001;75(6):1111-8.

12. The European Recombinant Human Chorionic Gonadotrophin Study Group. Induction of final follicular maturation and early luteinization in women undergoing ovulation induction for assisted reproduction treatment-recombinant HCG versus urinary HCG. Hum Reprod. 2000;15(7):1446-51.

13. Farrag A, Costantini A, Manna C, et al. Recombinant HCG for triggering ovulation increases the rate of mature oocytes in women treated for ICSI. J Assist Reprod Genet. 2008;25(9-10):461-6.

14. Uhler ML, Beltsos AN, Grotjan HE, et al. Age-matched comparison of recombinant and urinary $\mathrm{HCG}$ for final follicular maturation. Reprod Biomed Online. 2006;13(3):315-20.

15. Abdelmassih V, Oliveira FG, Goncalves SP, et al. A prospective, randomized and blinded comparison between 10, 000 IU urinary and $250 \mu \mathrm{g}$ recombinant human chorionic gonadotropin for oocyte maturation in in vitro fertilization cycles. J Assist Reprod Genet. 2005;22(4):149-53.

16. Kovacs P, Kovats T, Bernard A, et al. Comparison of serum and follicular fluid hormone levels with recombinant and urinary human chorionic gonadotropin during in vitro fertilization. Fertil Steril. 2008;90(6):2133-7. (Epub 2008 Jan 4).

17. Chan $\mathrm{CC}, \mathrm{Ng} \mathrm{EH}$, Tang OS, et al. A prospective, randomized, double-blind study to compare two doses of recombinant human chorionic gonadotropin in inducing final oocyte maturity and the hormonal profile during the luteal phase. J Clin Endocrinol Metab. 2005;90(7):3933-8. (Epub 2005 May 3).

18. Littman ED, Milki AA. The combination of urinary and recombinant HCG improves outcome in patients with decreased oocyte/ follicle ratio in previous cycles. Eur J Obstet Gynecol Reprod Biol. 2003;109(1):60-2.

19. Lorusso F, Palmisano M, Serratì G, et al. Intrauterine insemination with recombinant or urinary human chorionic gonadotropin: a prospective randomized trial. Gynecol Endocrinol. 2008;24(11): 644-8. 